\title{
Longitudinal Trajectories of Cognitive Decline among Older Adults with Cardiovascular Disease
}

\author{
Ozioma C. Okonkwo ${ }^{a}$ Ronald A. Cohen ${ }^{b}$ John Gunstad ${ }^{d}$ Geoffrey Tremont $^{\text {b, c }}$ \\ Michael L. Alosco ${ }^{c}$ Athena Poppas ${ }^{b}$ \\ ${ }^{a}$ Department of Neurology, Johns Hopkins University School of Medicine, Baltimore, Md., ${ }^{b}$ Department of \\ Psychiatry and Human Behavior, Brown Medical School, and ' Neuropsychology Program, Rhode Island Hospital, \\ Providence, R.I., and d Department of Psychology, Kent State University, Kent, Ohio, USA
}

\section{Key Words}

Cardiovascular disease - Vascular cognitive impairment •

Cognitive decline

\begin{abstract}
Background: The long-term course of cognitive impairments secondary to cardiovascular disease (CVD) is unclear. In this study, we prospectively investigated the temporal pattern, rate and hierarchy of cognitive decline attributable to CVD - a risk factor for the development of vascular cognitive impairment ( $\mathrm{VCl}$ ) - and examined the influence of cardiac surgery and heart failure on cognitive decline. Methods: A total of 172 older adults with CVD were administered a comprehensive battery of neuropsychological tests at study entry, and at 12 and 36 months thereafter. Random coefficient regressions were used to investigate the temporal course, rate and hierarchy of cognitive decline, as well as to examine the effect of heart failure (reported by $21 \%$ of the sample) and cardiac surgery (reported by $44 \%$ of the sample) on trajectories of cognitive change. Results: The course of decline in cognition was linear for language and attention-
\end{abstract}

executive function-psychomotor speed, and curvilinear for visuospatial abilities, memory and overall cognition. The decline in attention-executive function-psychomotor speed was smaller than the decline in other domains. The greatest decline occurred in visuospatial abilities. The rate of decline in cognition was not altered by a history of heart failure. Patients who had undergone cardiac surgery exhibited slower deceleration in their rates of decline in overall cognition. At baseline, patients with a history of heart failure had comparatively poorer attention-executive function-psychomotor speed, overall cognition and, to a lesser extent, visuospatial scores. Conclusion: There is measurable decline in neurocognitive function among patients with CVD. This decline is linear in some cognitive domains and curvilinear in others and is not attributable to the normal aging process. Cardiac surgery, but not heart failure, significantly affects the trajectory of cognitive decline. Because most vascular risk factors are modifiable, preventive measures such as lifestyle changes may be useful in retarding cognitive decline among patients with CVD, thus preventing the onset of $\mathrm{VCl}$.

Copyright $\odot 2010$ S. Karger AG, Basel

\section{KARGER}

두 2010 S. Karger AG, Basel

Fax +41613061234 E-Mail karger@karger.ch www.karger.com www.karger.com/ced
Ozioma C. Okonkwo

Department of Neurology, Johns Hopkins University School of Medicine 1620 McElderry Street, Reed Hall East 2

Baltimore, MD 21205 (USA)

Tel. +1 410502 2188, Fax +1 410502 2189, E-Mail ozioma@jhmi.edu 


\section{Introduction}

There has been a renewed interest in understanding the nature and extent of cognitive impairment secondary to vascular disease [1] as a result of the growing recognition that persons with cardiovascular disease (CVD) experience diminution in cognitive function even in the absence of major cardiac events or clinically relevant stroke [2]. CVD-associated cognitive impairments often impact everyday functional abilities [3-5], even when the impairments are not so severe as to incur a diagnosis of dementia $[6,7]$. Indeed, an emerging consensus in the field proposes that cognitive impairment attributable to vascular disorders should be viewed along a continuum, ranging from minimal cognitive difficulties (i.e. 'brain at risk') to frank dementia $[1,8,9]$.

While CVD-associated cognitive impairments vary as a function of the underlying etiology $[9,10]$, deficits in attention, executive functions, psychomotor speed and information processing are most common [10-13]. Impairment in these cognitive domains is presumed to be due to disruption of frontal and subcortical brain systems [14], with support for this position coming from studies that have demonstrated relationships between white matter lesions on neuroimaging and select cognitive deficits among persons with vascular diseases $[15,16]$. It is also well established that specific vascular factors (e.g. body mass index, serum cholesterol concentration and systolic blood pressure $[17,18]$ ) as well as some indices of vascular integrity (e.g. systemic perfusion, brachial artery reactivity and carotid intima-media thickness [19-23]) or metabolic function (e.g. C-reactive protein and brain natriuretic peptide [24-26]) are associated with cognitive performance in persons with CVD.

A question that has not received sufficient attention relates to the long-term course of cognitive impairments attributable to CVD. For example, whereas it is established that CVD is associated with select deficits in the attention-executive function-psychomotor speed domain, it remains unknown whether performance in this domain deteriorates more precipitously compared to other cognitive domains. Furthermore, we currently do not know whether cognitive decline in this population proceeds linearly (similar to the progressive decline observed in Alzheimer's disease), or whether some nonlinear trajectory obtains. Prior studies that have examined cognitive decline due to CVD have either used data from two time points (e.g. baseline and a follow-up period) $[24,27$ $29]$ or were restricted to patients who underwent coronary artery bypass graft (CABG) [30-32]. Therefore, their ability to provide information regarding the rate, hierarchy and temporal course of CVD-related cognitive decline, or regarding cognitive decline in the general population of patients with CVD, was limited.

In this study, we attempted to address these crucial knowledge gaps by investigating cognitive function over a 3 -year period in a cohort of ambulatory older adults with a variety of CVD, and by representing a wide range of cognitive functioning. Our primary aims were to determine (i) the temporal pattern of cognitive change, and (ii) whether cognitive change occurred comparably across all domains. Given that chronic CVD has shown to be linked to the development of vascular cognitive impairment (VCI) [6], and because cognitive decline in VCI likely does not occur in the same inexorably progressive manner as is seen in Alzheimer's disease, we expected to find a curvilinear course of cognitive change in this cohort. We expected to observed declines in the domain of attentionexecutive function-psychomotor speed though hypothesized that significant declines would also be evident across other domains, reflecting the impact of chronic CVD on global cognitive function. As secondary aims, we separately examined the effects of two cardiac factors - heart failure and CABG/heart valve surgery - on cognitive change in this cohort. We hypothesized that patients who had a history of these factors would exhibit a comparatively faster rate of decline in cognitive function.

\section{Patients and Methods}

\section{Participants}

The sample consisted of 172 community-dwelling individuals enrolled in a prospective study examining cognitive function among older adults with CVD. They were recruited from local hospitals, cardiac rehabilitation programs, cardiology practices, and by advertisement. To be enrolled in the study, participants had to be between 55 and 85 years old and have an established history of CVD including myocardial infarction, cardiac surgery, heart failure, atrial fibrillation and hypertension. Exclusion criteria included end-stage heart disease, history of traumatic brain injury with loss of consciousness exceeding $10 \mathrm{~min}$, neurological diseases (e.g. Parkinson's disease), current depressed mood, major psychiatric illness (e.g. bipolar disorder and schizophrenia) and MRI contraindications (e.g. pacemaker implant). Persons with Alzheimer's disease and other dementias (including vascular dementia) were also excluded. Of note, persons with a history of clinically manifest stroke were excluded from this study. Written informed consent was obtained from all participants as part of this study approved by the institutional review board. Table $1 \mathrm{de}-$ tails the characteristics of the participants at baseline. Of the 172 participants who completed the baseline evaluation, 106 subsequently had a 12-month follow-up evaluation, and 100 subsequently had a 36-month follow-up evaluation. 
Table 1. Characteristics of participants at baseline

\begin{tabular}{|c|c|}
\hline \multicolumn{2}{|l|}{ Demographic } \\
\hline Age, years & $69.18 \pm 7.61$ \\
\hline Female, $\%$ & 38.0 \\
\hline Ethnic minority, \% & 7.6 \\
\hline Years of education & $14.29 \pm 2.74$ \\
\hline BDI-II score & $5.34 \pm 4.22$ \\
\hline MMSE score & $28.47 \pm 1.67$ \\
\hline DRS-2 total score & $137.27 \pm 5.27$ \\
\hline \multicolumn{2}{|l|}{ Vascular indices } \\
\hline Systolic blood pressure, $\mathrm{mm} \mathrm{Hg}$ & $129.52 \pm 19.69$ \\
\hline Systolic blood pressure SD, mm Hg & $8.31 \pm 5.73$ \\
\hline Diastolic blood pressure, $\mathrm{mm} \mathrm{Hg}$ & $67.21 \pm 9.94$ \\
\hline Ejection fraction & $0.59 \pm 0.12$ \\
\hline Cardiac output, $1 / \mathrm{min}$ & $4.55 \pm 1.05$ \\
\hline C-reactive protein, $\mathrm{mg} / \mathrm{l}$ & $0.20 \pm 0.17$ \\
\hline BAR-reactive hyperemia, $\%$ change & $5.93 \pm 5.87$ \\
\hline $\mathrm{BAR}$ nitroglycerine, $\%$ change & $14.87 \pm 7.92$ \\
\hline Total brain volume ${ }^{\mathrm{a}}, \mathrm{cm}^{3}$ & $1,762.04 \pm 194.28$ \\
\hline Total $\mathrm{WMH}^{\mathrm{a}}, \mathrm{cm}^{3}$ & $11.17 \pm 18.68$ \\
\hline Total WMH percent $\mathrm{t}^{\mathrm{a}, \mathrm{b}}$ & $0.61 \pm 0.99$ \\
\hline \multicolumn{2}{|l|}{ Cardiovascular disease, $\%$} \\
\hline Hypertension & 72.9 \\
\hline Atrial fibrillation & 16.9 \\
\hline Heart failure & 20.7 \\
\hline Myocardial infarction & 45.9 \\
\hline Type 2 diabetes & 21.5 \\
\hline Hypercholesterolemia & 41.4 \\
\hline Cigarette smoking & 52.7 \\
\hline Heart valve surgery & 12.7 \\
\hline CABG & 37.0 \\
\hline
\end{tabular}

Values denote means \pm SD unless indicated otherwise. BDIII = Beck's Depression Inventory II; MMSE = Mini-Mental State Examination; DRS-2 = Dementia Rating Scale, 2nd edition; $\mathrm{BAR}=$ brachial artery response; $\mathrm{WMH}=$ white matter hyperintensity.

${ }^{a}$ MRI data were obtained from only 39 participants.

b Total WMH percent computed as (total WMH volume/ total brain volume) $\times 100$.

\section{Neuropsychological Assessment}

A comprehensive neuropsychological test battery was administered to all participants at each study visit. All tests were administered and scored by trained technicians, using standardized procedures and under the supervision of a licensed clinical neuropsychologist (R.A.C.). In addition to screening measures of global cognition (i.e. Mini-Mental State Examination, MMSE, and Dementia Rating Scale, 2nd edition, DRS-2), the neurocognitive battery assessed the domains of language, visuospatial abilities, memory and attention-executive function-psychomotor speed, using psychometric instruments with established reliabilities and validities [33]. Table 2 presents the specific tests used as well as the participants' test scores at baseline. Of note, as in our prior studies, the attention-executive function-psychomotor
Table 2. Neuropsychological test performance at baseline

\begin{tabular}{|c|c|c|}
\hline & Mean $\pm \mathrm{SD}$ & Range \\
\hline \multicolumn{3}{|l|}{ Language } \\
\hline Boston naming test & $54.74 \pm 5.11$ & $30-60$ \\
\hline Animal naming & $19.76 \pm 5.29$ & $8-36$ \\
\hline \multicolumn{3}{|l|}{ Visuospatial } \\
\hline Hooper visual organization test & $23.48 \pm 3.53$ & $11-30$ \\
\hline WAIS-III - block design & $32.13 \pm 11.05$ & $10-60$ \\
\hline CFT - copy & $30.53 \pm 5.64$ & $3-36$ \\
\hline \multicolumn{3}{|l|}{ Memory } \\
\hline CVLT-2 - trials $1-5$ & $45.09 \pm 11.65$ & $18-77$ \\
\hline CVLT-2 - short delay & $8.73 \pm 3.28$ & $1-15$ \\
\hline CVLT-2 - long delay & $8.90 \pm 3.48$ & $0-16$ \\
\hline CVLT-2 - recognition discriminability & $91.15 \pm 7.06$ & $61-100$ \\
\hline CFT - immediate recall & $14.43 \pm 7.58$ & $0-34$ \\
\hline CFT - delayed recall & $14.23 \pm 7.30$ & $0-34$ \\
\hline CFT - recognition discriminability & $19.53 \pm 2.28$ & $8-24$ \\
\hline BVMT-R - trials $1-3$ & $17.10 \pm 6.95$ & $1-36$ \\
\hline BVMT-R - delayed recall & $7.08 \pm 3.13$ & $0-12$ \\
\hline BVMT-R - recognition discriminability & $5.07 \pm 1.14$ & $0-6$ \\
\hline \multicolumn{3}{|c|}{ Attention-executive function-psychomotor speed } \\
\hline Trails A - time & $39.01 \pm 12.92$ & $16-93$ \\
\hline Trails B - time & $100.96 \pm 52.99$ & $34-300$ \\
\hline Letter search - time & $92.86 \pm 25.79$ & $49-200$ \\
\hline Letter search - errors & $1.56 \pm 2.36$ & $0-12$ \\
\hline Stroop test - interference trial; time & $30.88 \pm 9.47$ & $10-73$ \\
\hline COWAT & $40.18 \pm 12.66$ & $12-75$ \\
\hline \multicolumn{3}{|l|}{ Grooved pegboard - dominant hand; } \\
\hline WAIS-III - digit span & $17.46 \pm 3.72$ & $8-28$ \\
\hline WAIS-III - digit symbol & $54.94 \pm 14.46$ & $19-98$ \\
\hline
\end{tabular}

WAIS-III = Wechsler Adult Intelligence Scale, 3rd edition; CFT $=$ Rey-Osterrieth Complex Figure Test; CVLT-2 = California Verbal Learning Test, 2nd edition; BVMT-R = Brief Visual Memory Test, revised edition; COWAT $=$ Controlled Oral Word Association Test.

speed domain consisted of measures presumably mediated by frontal-subcortical systems [19, 21, 24-26, 29].

\section{Brain MRI Protocol and White Matter Hyperintensity \\ Quantification}

A small subset of the participants $(n=39)$ underwent brain MRI using a Siemens Symphony 1.5-tesla unit. This subgroup consisted of 14 women and 25 men with a mean age ( \pm SD) of 71.44 $( \pm 7.24)$ years, mean education of 13.92 ( \pm 2.16 ) years, mean MMSE score of 28.92 ( \pm 1.11), mean DRS-2 total score of 137.90 ( \pm 4.64), and a Beck Depression Inventory II score of 4.32 ( \pm 2.90 ). They differed significantly from those participants who did not undergo MRI regarding age (71.44 vs. 68.66 years; $\mathrm{p}=0.039$ ), MMSE (28.92 vs. 28.35; $\mathrm{p}=0.017)$, systolic blood pressure SD (11.18 
vs. $7.17 \mathrm{~mm} \mathrm{Hg}$; $<$ < 0.001) and history of diabetes (10.3 vs. $25.2 \%$; $\mathrm{p}=0.047$ ), but not regarding any other baseline characteristic.

The imaging protocol included axial $\mathrm{T}_{1^{-}}, \mathrm{T}_{2^{-}}$and FLAIR (fluidattenuated inversion recovery)-weighted images (field of view $=$ 24.7; matrix $=192 \times 256 ; \mathrm{TR} / \mathrm{TE}=6,000 / 105 ; 5$-mm-thick slices $/$ 2-mm gap). The FLAIR images were imported into Analyze 5.0 ${ }^{\circledR}$ (Biomedical Imaging Resource; Mayo Foundation, Rochester, Minn., USA), where the skull was stripped and the brain stem and cerebellum removed, leaving only brain parenchyma. Next, utilizing a semiautomated threshold method in Analyze, the total number of voxels representing white matter hyperintensities (WMH) were isolated from each slice and summed to calculate the participant's total WMH. Of note, each slice was visually reviewed by 2 trained raters and edited as needed. These raters achieved intraand interrater reliabilities of $>0.90$. Total $\mathrm{WMH}$ volume was normalized to total brain volume by using the formula: (total WMH volume/total brain volume) $\times 100$. This normalization yielded an index of total WMH load relative to the total amount of brain tissue and reflected the degree of total brain volume impacted by WMH.

\section{Data Analyses}

Participants' scores on the measures that constituted each neurocognitive domain were Z-transformed (using sample means and $S D$ at respective time points), summed and averaged to create a composite variable representing that domain at that time point. To ensure that higher scores on all the composites indicated better performance, $\mathrm{Z}$ scores for neuropsychological tests whose unit of measurement is completion time (e.g. trails A) were multiplied by -1 prior to the creation of the composite measures to correct for the fact that shorter completion time reflects better performance. In addition, a fifth composite measure, 'overall cognition', was created by summing and averaging participants' scores on each neurocognitive composite (i.e. language, visuospatial abilities, memory and attention-executive function-psychomotor speed). A similar procedure has been used in prior studies $[4,30]$.

To investigate the temporal course of change in cognitive function, we fitted random coefficient regressions that modeled participants' scores on each cognitive composite across the 3 time points under consideration [34]. Random coefficient regression, also called individual growth curve modeling, provides a flexible approach to analyzing longitudinal data. It easily accommodates variability in the number and spacing of assessment points across study participants $[30,34]$. In addition, because it assumes that each individual's observed scores are a random sample of data from their underlying true growth curve, it facilitates analyses that include all participants, even those with only one wave of data [34]. Of particular interest to our study, it allows an examination of temporal patterns in the data.

To determine whether cognitive change in our sample proceeded linearly or curvilinearly, we fitted 2 sets of models for each cognitive domain. In one set, we only tested a linear term for time (i.e. year), whereas in the other set, we included linear (i.e. year) and quadratic (i.e. year $\times$ year) terms for time. The year effect from the linear models would reveal the annual rate of change in cognitive function, whereas the year $\times$ year effect from the quadratic model would indicate the rate of acceleration or deceleration in cognitive change over time. Additionally, for both linear and quadratic models, we first tested a model that only adjusted for baseline performance on the cognitive domain being examined and then tested another model that adjusted for baseline per- formance and age. Given that the linear models are nested within the quadratic models, in addition to examining the $\mathrm{p}$ value of the year $x$ year terms so as to determine whether a quadratic model fit the data, we also performed deviance tests (i.e. -2LL linear model minus -2LL quadratic model) to ascertain the incremental fit of each quadratic model relative to its corresponding, more parsimonious linear model. Because all neurocognitive composites are on the same metric (i.e. $\mathrm{Z}$ scores), the magnitude and sign of their regression estimates would indicate whether change occurred comparably across all neurocognitive domains.

The polynomial specification for time (i.e. linear or quadratic) that emerged as optimal for each cognitive domain was subsequently employed in further random coefficient regressions that examined the effects of 2 cardiac factors - major cardiac surgery (i.e. CABG or heart valve surgery) and heart failure - on change in cognition. In these latter models, we fitted terms for (i) age, baseline performance on the cognitive domain, cardiac factor, year and cardiac factor $\times$ year when the optimal polynomial specification for time was linear, or (ii) age, baseline performance, cardiac factor, year, year $x$ year, cardiac factor $x$ year and cardiac factor $x$ year $x$ year when the optimal polynomial specification for time was quadratic. For these models, the primary term of interest was the highest-order group-by-time interaction (i.e. cardiac factor $\times$ year for the linear models and cardiac factor $\times$ year $\times$ year for the quadratic models) because it would indicate whether those with a history of the cardiac factor - say, heart failure - have a significantly different change trajectory compared to those without such a history. Specifically, for the linear models, cardiac factor $\times$ year would reveal the estimated difference in annual rate of change in cognition between patients with and without a history of the factor under study, whereas for the quadratic models, cardiac factor $\times$ year $x$ year would reveal the estimated difference in rate of acceleration or deceleration in cognitive change between patients with and without a history of the cardiac factor under investigation.

All analyses were performed using SPSS 16.0 (SPSS Inc., Chicago, Ill., USA). Only findings with a two-tailed $\mathrm{p} \leq 0.05$ were considered significant.

\section{Results}

\section{Analysis of Attrition}

The 72 patients who failed to complete the 36 -month evaluation withdrew for various reasons, the most common being a worsening of their CVD (which affected their ability to participate actively in the study) and death. Analyses of baseline scores between those who completed the study and those who withdrew revealed that the withdrawers had significantly poorer language, visuospatial, overall cognition and MMSE scores compared to the completers. The withdrawers also tended to perform poorer on memory. In addition, they had significantly less years of education, and significantly higher baseline serum C-reactive protein. No other group differences emerged in the variables examined. These results are presented in table 3 . 
Table 3. Group differences at baseline: patients who completed the study versus those who withdrew

\begin{tabular}{|c|c|c|c|}
\hline & $\begin{array}{l}\text { Completers } \\
(\mathrm{n}=100)\end{array}$ & $\begin{array}{l}\text { Withdrawers } \\
(\mathrm{n}=72)\end{array}$ & $\mathrm{p}$ \\
\hline \multicolumn{4}{|l|}{ Cognitive composites } \\
\hline Language & $0.14 \pm 0.80$ & $-0.16 \pm 0.87$ & 0.022 \\
\hline Visuospatial & $0.15 \pm 0.66$ & $-0.17 \pm 0.80$ & 0.005 \\
\hline Memory & $0.08 \pm 0.68$ & $-0.11 \pm 0.66$ & 0.068 \\
\hline \multicolumn{4}{|c|}{ Attention-executive functions-psychomotor } \\
\hline speed & $0.06 \pm 0.55$ & $-0.07 \pm 0.65$ & 0.183 \\
\hline Overall cognition & $0.11 \pm 0.52$ & $-0.14 \pm 0.56$ & 0.004 \\
\hline \multicolumn{4}{|l|}{ Demographics } \\
\hline Age, years & $69.05 \pm 7.29$ & $69.26 \pm 7.83$ & 0.857 \\
\hline Female, \% & 33.3 & 42.5 & 0.221 \\
\hline Ethnic minority, \% & 4.1 & 14.6 & 0.171 \\
\hline Years of education & $14.81 \pm 2.73$ & $13.59 \pm 2.56$ & 0.004 \\
\hline BDI-II & $5.12 \pm 4.22$ & $5.76 \pm 4.30$ & 0.435 \\
\hline MMSE & $28.70 \pm 1.45$ & $28.16 \pm 1.90$ & 0.039 \\
\hline DRS-2 total score & $137.88 \pm 4.74$ & $136.78 \pm 5.07$ & 0.142 \\
\hline \multicolumn{4}{|l|}{ Vascular indices } \\
\hline Systolic blood pressure, $\mathrm{mm} \mathrm{Hg}$ & $130.74 \pm 18.18$ & $127.77 \pm 23.09$ & 0.409 \\
\hline Systolic blood pressure SD, mm Hg & $7.95 \pm 4.57$ & $8.88 \pm 7.67$ & 0.375 \\
\hline Diastolic blood pressure, $\mathrm{mm} \mathrm{Hg}$ & $67.40 \pm 8.79$ & $67.13 \pm 11.95$ & 0.878 \\
\hline Ejection fraction & $0.58 \pm 0.11$ & $0.59 \pm 0.13$ & 0.938 \\
\hline Cardiac output, $1 / \mathrm{min}$ & $4.53 \pm 0.98$ & $4.62 \pm 1.17$ & 0.650 \\
\hline C-reactive protein, mg/l & $0.16 \pm 0.13$ & $0.26 \pm 0.20$ & 0.001 \\
\hline BAR-reactive hyperemia, \% change & $5.75 \pm 5.88$ & $6.12 \pm 6.17$ & 0.793 \\
\hline BAR nitroglycerine, $\%$ change & $14.61 \pm 8.22$ & $15.69 \pm 7.65$ & 0.595 \\
\hline Total brain volume ${ }^{\mathrm{a}}, \mathrm{cm}^{3}$ & $1,809.37 \pm 170.37$ & $1,663.73 \pm 210.41$ & 0.024 \\
\hline Total $\mathrm{WMH}^{\mathrm{a}}, \mathrm{cm}^{3}$ & $9.97 \pm 13.02$ & $13.66 \pm 27.50$ & 0.565 \\
\hline Total WMH percent ${ }^{\mathrm{a}, \mathrm{b}}$ & $0.54 \pm 0.71$ & $0.76 \pm 1.43$ & 0.516 \\
\hline \multicolumn{4}{|l|}{ Cardiovascular disease, $\%$} \\
\hline Hypertension & 73.7 & 74.0 & 0.972 \\
\hline Atrial fibrillation & 15.6 & 20.4 & 0.479 \\
\hline Heart failure & 16.5 & 26.8 & 0.145 \\
\hline Myocardial infarction & 51.5 & 39.7 & 0.125 \\
\hline Type 2 diabetes & 20.2 & 23.3 & 0.626 \\
\hline Hypercholesterolemia & 46.5 & 37.0 & 0.214 \\
\hline Cigarette smoking & 53.7 & 51.2 & 0.792 \\
\hline Heart valve surgery & 12.9 & 13.3 & 0.945 \\
\hline CABG & 37.1 & 39.1 & 0.792 \\
\hline
\end{tabular}

\section{Temporal Pattern and Rate of Change in Cognitive Function}

The random coefficient regressions revealed both linear and curvilinear patterns of change in cognitive function (fig. 1). The models that examined change in cognition as a function of linear time revealed that, as a group, the study participants experienced significant declines in all cognitive domains and on overall cognition. These declines ranged from 0.02 to 0.05 of an SD per year. When the analyses were repeated while adjusting for age (in addition to the original adjustment for baseline performance on the cognitive domain), results remained virtu- 
Table 4. Growth curve models of temporal course and rate of change in cognitive function

\begin{tabular}{|c|c|c|c|c|c|c|c|c|}
\hline \multirow[t]{2}{*}{ Domain } & \multicolumn{4}{|c|}{ Linear effect ${ }^{1}$} & \multicolumn{4}{|c|}{ Quadratic effect ${ }^{2}$} \\
\hline & estimate & $95 \% \mathrm{CI}$ & $\mathrm{p}^{3}$ & $\mathrm{p}^{4}$ & estimate & $95 \%$ CI & $\mathrm{p}^{3}$ & $\mathrm{p}^{4}$ \\
\hline Language & $-0.05(0.02)$ & $-0.08,-0.02$ & 0.004 & 0.004 & $0.02(0.02)$ & $-0.02,0.06$ & 0.318 & 0.347 \\
\hline Visuospatial abilities & $-0.05(0.02)$ & $-0.08,-0.02$ & 0.002 & 0.002 & $0.06(0.02)$ & $0.02,0.10$ & 0.007 & 0.014 \\
\hline Memory & $-0.03(0.01)$ & $-0.05,-0.01$ & 0.012 & 0.013 & $0.04(0.01)$ & $0.01,0.06$ & 0.013 & 0.023 \\
\hline $\begin{array}{l}\text { Attention-executive functions- } \\
\text { psychomotor speed }\end{array}$ & $-0.02(0.01)$ & $-0.04,-0.00$ & 0.024 & 0.026 & $0.02(0.01)$ & $-0.01,0.04$ & 0.130 & 0.167 \\
\hline Overall cognition & $-0.04(0.01)$ & $-0.05,-0.02$ & $<0.001$ & $<0.001$ & $0.03(0.01)$ & $0.01,0.05$ & $<0.001$ & 0.001 \\
\hline
\end{tabular}

Values in parentheses denote SE.

${ }^{1}$ Linear effects were derived from models that tested only a 'year' term for time. Therefore, these effects represent annual rates of decline.

${ }^{2}$ Quadratic effects were derived from models that simultaneously tested linear (i.e. year) and quadratic (i.e. year $\times$ year) terms for time. Because the quadratic terms are the effects of interest, test statistics for the linear terms are not reported in the table. The reported quadratic terms indicate the rate of deceleration in cognitive decline over time.

${ }^{3} \mathrm{p}$ from model that only adjusted for baseline performance.

${ }^{4} \mathrm{p}$ from model that adjusted for baseline performance and age.

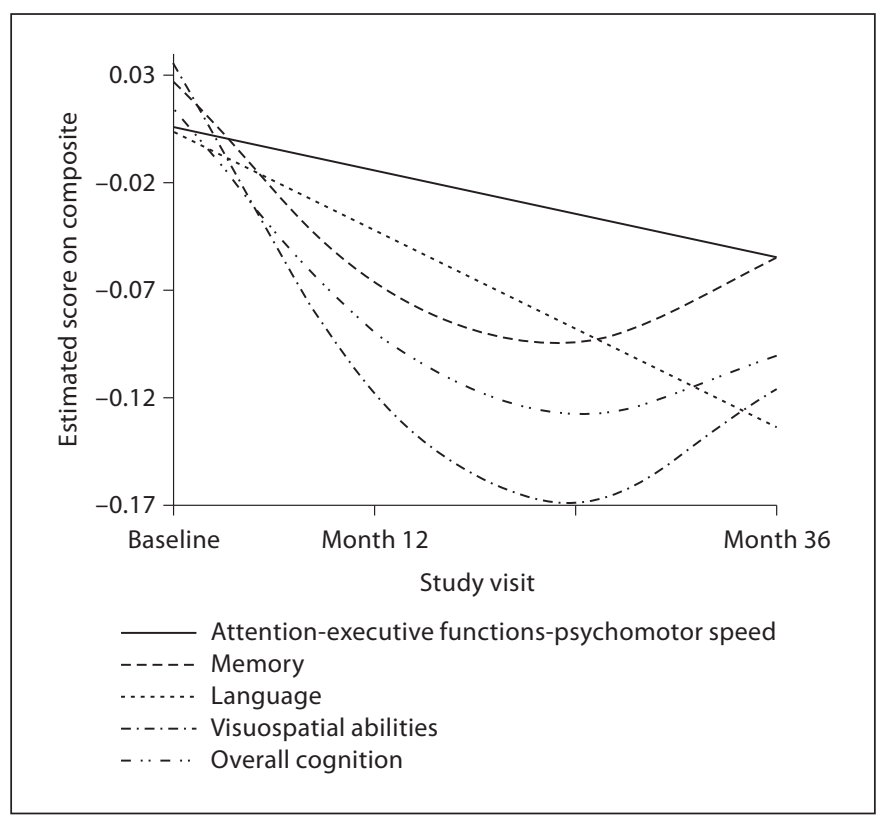

Fig. 1. Temporal patterns of change in cognitive function across time. The regression lines for language and attention-executive function-psychomotor speed were derived from linear models, whereas those for visuospatial abilities, memory and overall cognition were derived from quadratic models.

ally unchanged. On the other hand, the models that treated time as a quadratic function revealed significant decelerations in the rate of cognitive decline for visuospatial abilities, memory and overall cognition, which ranged from 0.03 to 0.06 of an SD. After additional adjustment for age, these decelerations persisted. These results are presented in table 4. Deviance tests, using -2LL values from the baseline- and age-adjusted models, were conducted to ascertain that quadratic time truly provided better fit to the visuospatial, memory and overall cognition data, relative to the more parsimonious linear time. These tests confirmed the superiority of the quadratic time effect: visuospatial abilities $\left[\chi^{2}(1)=5.90 ; p=0.015\right]$, memory $\left[\chi^{2}(1)=5.17 ; \mathrm{p}=0.023\right]$ and overall cognition $\left[\chi^{2}(1)=11.45 ; \mathrm{p}<0.001\right]$.

\section{Hierarchy of Change across Cognitive Domains}

Examination of the estimates from the linear time random coefficient regressions reported above revealed that the rate of decline in the attention-executive functions-psychomotor speed domain was lower than the decline in other cognitive domains. The greatest decline was observed in the visuospatial domain (barring rounding) (table 4). However, as can be appreciated from the 95\% confident limits reported in table 4, there was considerable overlap in the CI of the domains' slopes, making it difficult to assert that any domain declined significantly faster or slower than others did.

\section{Change in Cognition as a Function of Cardiac Factors}

Based on the deviance tests reported above, quadratic time was deemed optimal for visuospatial abilities, memory and overall cognition, whereas linear time appeared more fitting for the other domains. Table 5 details the results of the random coefficient regressions that examined the effect of major cardiac surgery (which was reported 
Table 5. Effect of cardiac factors on trajectories of change in cognition

\begin{tabular}{|c|c|c|c|c|c|c|}
\hline & \multicolumn{6}{|c|}{ Major cardiac surgery ${ }^{1}$} \\
\hline & \multicolumn{3}{|c|}{ linear interaction $^{2}$} & \multicolumn{3}{|c|}{ quadratic interaction $^{3}$} \\
\hline & estimate & SE & $\mathrm{p}$ & estimate & SE & $\mathrm{p}$ \\
\hline Language & 0.02 & 0.03 & 0.568 & - & - & - \\
\hline Visuospatial abilities & - & - & - & -0.05 & 0.04 & 0.217 \\
\hline Memory & - & - & - & -0.02 & 0.03 & 0.409 \\
\hline $\begin{array}{l}\text { Attention-executive functions- } \\
\text { psychomotor speed }\end{array}$ & 0.00 & 0.02 & 0.865 & - & - & - \\
\hline \multirow[t]{4}{*}{ Overall cognition } & - & - & - & -0.04 & 0.02 & 0.037 \\
\hline & \multicolumn{6}{|c|}{ Heart failure } \\
\hline & \multicolumn{3}{|c|}{ linear interaction ${ }^{2}$} & \multicolumn{3}{|c|}{ quadratic interaction $^{3}$} \\
\hline & estimate & SE & $\mathrm{p}$ & estimate & SE & $\mathrm{p}$ \\
\hline Language & 0.01 & 0.05 & 0.851 & - & - & - \\
\hline Visuospatial abilities & - & - & - & -0.04 & 0.06 & 0.452 \\
\hline Memory & - & - & - & -0.01 & 0.04 & 0.860 \\
\hline $\begin{array}{l}\text { Attention-executive functions- } \\
\text { psychomotor speed }\end{array}$ & 0.02 & 0.03 & 0.479 & - & - & - \\
\hline Overall cognition & - & - & - & -0.02 & 0.03 & 0.484 \\
\hline
\end{tabular}

For all analyses, the reference group is patients without history of the cardiac factor under investigation (i.e. major cardiac surgery or heart failure).

${ }^{1}$ Major cardiac surgery was defined as either CABG or heart valve surgery.

${ }^{2}$ Major cardiac surgery/heart failure $\times$ year. Linear interactions were obtained from models that tested the following terms: year, cardiac factor, baseline performance on the cognitive domain being modeled, age and cardiac factor $\times$ year. The estimates reported are for the cardiac factor $\times$ year terms, which represent the estimated difference in annual rate of decline in cognition be- tween patients with a history of the cardiac factor under investigation and those without such a history.

${ }^{3}$ Major cardiac surgery/heart failure $\times$ year $\times$ year. Quadratic interactions were obtained from models that tested the following terms: year, year $\times$ year, cardiac factor, baseline performance on the cognitive domain being modeled, age, cardiac factor $\times$ year and cardiac factor $\times$ year $\times$ year. The estimates reported are for the cardiac factor $\times$ year $\times$ year terms, which represent the estimated difference in the rate of deceleration in cognitive change between patients with a history of the cardiac factor under investigation and those without such a history. by $44 \%$ of the sample) on change trajectory. The only significant difference in the trajectories of those with a history of cardiac surgery relative to those without such a history occurred on overall cognition. Whereas those without a history of cardiac surgery experienced a deceleration of 0.05 in the rate of decline in overall cognition (not shown in table 5), those who had undergone cardiac surgery experienced a deceleration of 0.01 (i.e. 0.05-0.04). This finding is illustrated in figure 2 . Because these random coefficient regressions adjusted for baseline performance in respective cognitive domains, they did not permit an assessment of the effect of cardiac surgery on cognitive performance at baseline. Therefore, we performed an exploratory multivariate analysis of variance, adjusting for age, to examine whether there were any differ- ences at baseline between patients with and without a history of cardiac surgery. This analysis failed to find any multivariate (or univariate) difference between these 2 patient groups at baseline: $\lambda=0.96, F(4,166)=1.76, \mathrm{p}=$ 0.139 .

Table 5 also details the results of the analyses that examined the effect of heart failure (reported by $21 \%$ of the sample) on change trajectory. There were no differences in the trajectories of those with a history of heart failure compared to those without such a history. We then performed an exploratory multivariate analysis of variance, adjusting for age, to examine the effect of heart failure on cognitive performance at baseline. This analysis revealed a significant multivariate difference between patients with and without a history of heart failure: $\lambda=0.92$, 




Fig. 2. Effect of major cardiac surgery on change in overall cognition.

$\mathrm{F}(4,130)=3.03, \mathrm{p}=0.020$. An examination of univariate effects revealed that patients with a history of heart failure had significantly lower baseline scores on attentionexecutive functions-psychomotor speed (estimate: -0.38 ; SE: $0.11 ; p<0.001$ ) and overall cognition (estimate: -0.24 ; SE: $0.11 ; p=0.027)$ relative to those without such a history. They also tended to have lower baseline visuospatial scores (estimate: -0.27 ; SE: $0.16 ; \mathrm{p}=0.087$ ).

\section{Exploratory Examination of Change in Cognition as a} Function of Total WMH

To determine whether total WMH load had any impact on cognitive decline, we performed exploratory random effect analyses that regressed longitudinal scores on each cognitive domain (1) on age, baseline performance on the cognitive domain, year, total $\mathrm{WMH}$ and total $\mathrm{WMH} \times$ year (for those domains wherein linear time was optimal), or (2) on age, baseline performance, year, total $\mathrm{WMH}$, year $\times$ year, total $\mathrm{WMH} \times$ year and total $\mathrm{WMH} \times$ year $\times$ year (for those domains wherein quadratic time was optimal). These analyses revealed that total WMH was not a significant predictor of decline on any cognitive domain within the subset $(\mathrm{n}=39)$ of subjects who had imaging data (language: total WMH $\times$ year $=-0.01, \mathrm{SE}=0.03, \mathrm{p}=0.887$; attention-executive functions-psychomotor speed: total $\mathrm{WMH} \times$ year $=$ $0.02, \mathrm{SE}=0.02, \mathrm{p}=0.340$; visuospatial abilities: total $\mathrm{WMH} \times$ year $\times$ year $=0.06, \mathrm{SE}=0.04, \mathrm{p}=0.178 ; \mathrm{mem}-$ ory: total $\mathrm{WMH} \times$ year $\times$ year $=-0.02, \mathrm{SE}=0.03, \mathrm{p}=$ 0.536 ; overall cognition: total WMH $\times$ year $\times$ year $=$ $0.01, \mathrm{SE}=0.02, \mathrm{p}=0.609$ ).

\section{Discussion}

Although there is increased recognition that older adults with vascular risk factors or diseases experience cognitive impairments even in the absence of stroke or other major cardiac events $[1,2,17,18,21]$, there has not been a systemic attempt to prospectively investigate the cognitive outcome of these persons. Accordingly, in this study, we examined change in cognition among a cohort of older adults with a wide range of CVD as well as attendant cognitive dysfunction. To our knowledge, this study represents the first attempt to address this important issue.

Our primary aims were to characterize the temporal course of CVD-associated change in cognitive function and to determine whether cognitive change occurred at the same rate across all domains. Our results demonstrated that cognitive change occurred in a linear fashion in some domains (language and attention-executive functions-psychomotor speed) and curvilinearly in others (visuospatial abilities, memory and overall cognition). Although it is not entirely clear why decline was linear in some cognitive domains and curvilinear in other domains, a plausible hypothesis is that the curvilinear trajectories represent a gradual recovery of cognitive functioning as a result of intensive treatment. If that is the case, then a longer follow-up of this cohort (in the context of ongoing treatment) might also reveal curvilinearity in the currently linear trajectories for language and attention-executive functions-psychomotor speed.

Inspection of the parameter estimates from the models that specified a linear function of time indicated that decline in attention-executive-psychomotor functions occurred slower than decline in other cognitive domains. This finding is somewhat counterintuitive, given that past cross-sectional research indicates that various vascular abnormalities (e.g. reduced ejection fraction and cardiac output, impaired brachial artery reactivity, carotid artery thickening and elevated C-reactive protein) impair attention-executive functions-psychomotor speed but not other cognitive functions [19-24]. Even so, there is equal evidence that patients with chronic CVD show impairments in other cognitive domains besides attention-executive functions-psychomotor speed [6, 35-37]. Indeed, the term 'dementia' in vascular dementia presup- 
poses impairments in multiple cognitive domains. Accordingly, our current finding of comparatively higher rates of decline in language, visuospatial abilities and memory suggests that while vascular integrity may be most strongly associated with attention-executive functions-psychomotor speed at any given point in time, the cumulative effect of CVD over time results in more generalized cognitive impairment. It is important to note that the cognitive decline observed in this cohort is not simply attributable to the patients' aging as the decrements persisted even after adjusting for age in the models. In addition, the significant curvilinear change trajectories provide further support for the view that observed declines are not likely proxies for aging, given that age proceeds linearly.

A handful of studies have previously examined change in cognition as a result of vascular alterations $[24,27,28]$. Hoth et al. [24] reported that, over a 1-year period, CVD patients demonstrated worsening of constructional praxis, but improvements in visual scanning and immediate verbal recall. Another study that was also conducted over a 1-year span [27] found that patients with VCI exhibited improvements in verbal learning and recall, and decrements in processing speed. In the Canadian Study of Health and Aging [28], there was evidence of a significant decline in global cognition among persons classified as having 'VCI - no dementia' at both study entry and 5 years later, indicating that deterioration in cognition occurs even in the absence of progression to a severer diagnostic status (i.e. dementia). As noted earlier, these studies are somewhat limited by the use of only two time points. Nonetheless, their findings are important for providing initial information regarding the evolution of CVD-related cognitive dysfunction.

Given current interest in the potential contributions of reduced cardiac output $[19,21,38-41]$ and major cardiac surgery [31, 32, 42-44] to cognitive decline among patients with CVD, we examined the association between history of heart failure or CABG/heart valve surgery and cognitive decline over the duration of the study. Our analyses failed to find a significant association between heart failure and rate of decline on any of the cognitive domains tested. Even so, we found that patients with heart failure had comparatively lower baseline scores on the attention-executive functions-psychomotor speed, overall cognition and, to a lesser extent, visuospatial domains. The apparent preservation of cognitive function over time among patients with heart failure might be due to the fact that many of them were receiving intense treatment including placement of ventricular stimulators, which shored up their cognition. Our findings are consonant overall with results from prior studies that investigated differences in cognitive performance between persons with and without a history of heart failure [40, 41, $45,46]$. Possible mechanisms for cognitive dysfunction in heart failure include systemic hypoperfusion $[19,20,40$, 45,47 ], cerebral hypoperfusion [39], cardiogenic emboli [41, 48], cerebral infarcts [39], biochemical alterations (e.g. in serum concentrations of renin, aldosterone, sodium and potassium) $[49,50]$ and endothelial abnormalities $[21,51,52]$.

The sole association between history of major cardiac surgery and rate of decline in cognition emerged in overall cognition, wherein patients who had undergone cardiac surgery showed a slower rebound in performance compared to CVD patients who had not undergone surgery. This finding suggests that cardiac surgery in this cohort had some effect on change in cognitive function beyond that due to the vascular disease itself. The literature on the effects of cardiac surgery on cognition is mixed. Whereas some studies report that cardiac surgery impairs cognition [31,32], others have found inconsistent [42] or no evidence of such impairment [30,53]. The reasons for this variability are not entirely clear. Although surgery-related cognitive dysfunction is often believed to be iatrogenic, this presumption has not been borne out by empirical studies $[31,53]$. Similarly, postoperative cognitive impairments have been shown to be unrelated perioperative strokes [44]. The lack of unequivocal associations between cardiac surgery and postoperative cognitive dysfunction may be consistent with the idea that cognitive decline following cardiac surgery is not due to surgery per se or surgery-related complications, but rather due to a progression of the underlying vascular diseases [30]. Clearly, further studies are warranted to better understand the dynamics of the association between cardiac surgery and prospective cognitive function.

Over the past decade, the relationship between cerebrovascular disease (typically quantified as amount of WMH on MRI scans) and cognitive impairment has received great attention $[7,15,54,55]$, and several groups, including ours, have reported that WMH load is associated with cognitive decline $[16,56,57]$. However, in the subset of participants who had MRI data in this study, we failed to find any associations between total $\mathrm{WMH}$ and rate of cognitive decline. It is possible that our negative findings are primarily due to our small sample size. Our findings may also suggest that in this subsample, cognitive decline was primarily a result of other factors associated with chronic CVD (e.g. the effects of chronic and 
general cerebral hypoperfusion) and of related complications rather than solely of white matter damage attributable to microvascular disease.

It is worth noting that patients who failed to complete the entire 36 months of the study had poorer baseline cognitive performance, less education and, for the most part, greater cardiovascular burden, particularly as indexed by serum C-reactive protein levels. It is theoretically plausible that patients who are more impaired at baseline would experience greater declines over time compared to those who are more intact. Accordingly, the worsening of cognitive performance over time that was observed in this study may actually underestimate the actual cognitive change that occurred in the original sample as a whole. The finding that withdrawers had comparatively worse cognitive functioning at baseline also underscores a potentially important clinical consideration when monitoring functional outcome among patients with CVD. Patients who show the greatest vulnerability to functional decline may be the most likely to be lost to follow-up such that their health care providers are unaware of their deteriorating functional status. It might be beneficial to direct particular effort toward developing effective ways of keeping these patients involved in ongoing health care.

A potential limitation of this study stems from the fact that the cohort consisted of a heterogeneous sample of older adults with CVD of varying severity. This issue primarily impacts the interpretation of the effects of specific cardiovascular factors such as heart failure or cardiac surgery. The parent study was not designed to examine the influence of a specific cardiovascular etiological factor such as cardiac surgery. Rather, its goal was to investigate how specific vascular physiological alterations such as reduced cardiac output, blood pressure variability and arterial stiffening influence cognitive outcome. In this regard, heterogeneity was necessary if the study's aims were to be feasibly tested. On the other hand, this heterogeneity has the benefit of making our sample more representative of the population of older adults with CVD, thus making it more likely that our findings will be generalized to other clinical settings. Another limitation is that most of these patients were receiving treatment for their vascular diseases. The extent to which our findings are affected by this fact is uncertain. The relative ethnic homogeneity of this cohort (primarily Caucasian) may also represent a limitation insofar as our findings may not replicate within a more ethnically diverse cohort. Finally, we only tested a specific nonlinear function of time. It is possible that a different curvilinear function of time (e.g. logarithmic) would have fit the data just as well, potentially leading to alternative conclusions regarding the temporal course of cognitive change in this cohort.

Despite these limitations, this study is unique in its prospective examination of the rate, hierarchy and temporal pattern of cognitive change among older adults with a variety of CVD - and exhibiting a range of baseline cognitive functioning from fairly intact to marked deficits - using an extensive neurocognitive battery. Therefore, it stands to make important contributions to the growing literature on vascular-related cognitive dysfunction. Future studies from our group will examine the demographic, systemic, vascular and neuroimaging predictors of change in cognition in this heterogeneous cohort of ambulatory older adults with CVD.

\section{Acknowledgment}

This research was supported by grant R01 AG017975 from the National Institute on Aging, USA.

\section{References}

1 Rao R, Howard R: Vascular dementia: dead or alive? Int J Geriatr Psychiatry 1998;13: 277-284.

$\checkmark 2$ Duron E, Hanon O: Vascular risk factors, cognitive decline, and dementia. Vasc Health Risk Manag 2008;4:363-381.

3 Leblanc GG, Meschia JF, Stuss DT, Hachinski V: Genetics of vascular cognitive impairment: the opportunity and the challenges. Stroke 2006;37:248-255.
4 Cohen RA, Moser DJ, Clark MM, Aloia MS, Cargill BR, Stefanik S, Albrecht A, Tilkemeier P, Forman DE: Neurocognitive functioning and improvement in quality of life following participation in cardiac rehabilitation. Am J Cardiol 1999;83:1374-1378.

$\checkmark 5$ Rockwood K, Wentzel C, Hachinski V, Hogan DB, MacKnight C, McDowell I: Prevalence and outcomes of vascular cognitive impairment. Vascular Cognitive Impairment Investigators of the Canadian Study of Health and Aging. Neurology 2000;54:447451.

\footnotetext{
6 Hachinski V, Iadecola C, Petersen RC, Breteler MM, Nyenhuis DL, Black SE, Powers WJ, DeCarli C, Merino JG, Kalaria RN, Vinters HV, Holtzman DM, Rosenberg GA, Wallin A, Dichgans M, Marler JR, Leblanc GG: National Institute of Neurological Disorders and Stroke-Canadian Stroke Network vascular cognitive impairment harmonization standards. Stroke 2006;37:2220-2241.
} 
-7 O’Brien JT, Erkinjuntti T, Reisberg B, Roman G, Sawada T, Pantoni L, Bowler JV, Ballard C, DeCarli C, Gorelick PB, Rockwood K, Burns A, Gauthier S, DeKosky ST: Vascular cognitive impairment. Lancet Neurol 2003; 2:89-98.

78 Desmond DW: The neuropsychology of vascular cognitive impairment: is there a specific cognitive deficit? J Neurol Sci 2004;226: 3-7.

\$9 Hachinski V: Vascular dementia: a radical redefinition. Dementia 1994;5:130-132.

10 O’Brien JT: Vascular cognitive impairment. Am J Geriatr Psychiatry 2006;14:724-733.

- 11 Sachdev PS, Brodaty H, Valenzuela MJ, Lorentz L, Looi JC, Wen W, Zagami AS: The neuropsychological profile of vascular cognitive impairment in stroke and TIA patients. Neurology 2004;62:912-919.

12 Garrett KD, Browndyke JN, Whelihan W, Paul RH, DiCarlo M, Moser DJ, Cohen RA, Ott BR: The neuropsychological profile of vascular cognitive impairment - no dementia: comparisons to patients at risk for cerebrovascular disease and vascular dementia. Arch Clin Neuropsychol 2004;19:745-757.

13 Nyenhuis DL, Gorelick PB, Geenen EJ, Smith CA, Gencheva E, Freels S, DeToledo-Morrell $\mathrm{L}$ : The pattern of neuropsychological deficits in Vascular Cognitive Impairment-No Dementia (Vascular CIND). Clin Neuropsychol 2004;18:41-49.

14 Alexander GE, DeLong MR, Strick PL: Parallel organization of functionally segregated circuits linking basal ganglia and cortex. Annu Rev Neurosci 1986;9:357-381.

15 Pantoni L, Poggesi A, Inzitari D: Cognitive decline and dementia related to cerebrovascular diseases: some evidence and concepts. Cerebrovasc Dis 2009;27(suppl 1):191-196.

-16 Tate DF, Jefferson AL, Brickman AM, Hoth KF, Gunstad J, Bramley K, Paul RH, Poppas A, Cohen RA: Regional white matter signal abnormalities and cognitive correlates among geriatric patients with treated cardiovascular disease. Brain Imaging Behav 2008; 2:200-206.

- 17 Paglieri C, Bisbocci D, Caserta M, Rabbia F, Bertello C, Canade A, Veglio F: Hypertension and cognitive function. Clin Exp Hypertens 2008;30:701-710.

18 Kivipelto M, Ngandu T, Fratiglioni L, Viitanen M, Kareholt I, Winblad B, Helkala EL, Tuomilehto J, Soininen H, Nissinen A: Obesity and vascular risk factors at midlife and the risk of dementia and Alzheimer disease. Arch Neurol 2005;62:1556-1560.

19 Jefferson AL, Poppas A, Paul RH, Cohen RA: Systemic hypoperfusion is associated with executive dysfunction in geriatric cardiac patients. Neurobiol Aging 2007;28:477-483.

$\checkmark 20$ Jerskey BA, Cohen RA, Jefferson AL, Hoth KF, Haley AP, Gunstad JJ, Forman DE, Sweet LH, Poppas A: Sustained attention is associated with left ventricular ejection fraction in older adults with heart disease. J Int Neuropsychol Soc 2009;15:137-141.
21 Cohen RA, Poppas A, Forman DE, Hoth KF, Haley AP, Gunstad J, Jefferson AL, Tate DF, Paul RH, Sweet LH, Ono M, Jerskey BA, Gerhard-Herman M: Vascular and cognitive functions associated with cardiovascular disease in the elderly. J Clin Exp Neuropsychol 2008;31:96-110.

22 Haley AP, Forman DE, Poppas A, Hoth KF, Gunstad J, Jefferson AL, Paul RH, Ler AS, Sweet LH, Cohen RA: Carotid artery intimamedia thickness and cognition in cardiovascular disease. Int J Cardiol 2007;121:148154.

-23 Haley AP, Sweet LH, Gunstad J, Forman DE, Poppas A, Paul RH, Tate DF, Cohen RA: Verbal working memory and atherosclerosis in patients with cardiovascular disease: an fMRI study. J Neuroimaging 2007;17:227233.

24 Hoth KF, Haley AP, Gunstad J, Paul RH, Poppas A, Jefferson AL, Tate DF, Ono M, Jerskey BA, Cohen RA: Elevated C-reactive protein is related to cognitive decline in older adults with cardiovascular disease. J Am Geriatr Soc 2008;56:1898-1903.

25 Gunstad J, Poppas A, Smeal S, Paul RH, Tate DF, Jefferson AL, Forman DE, Cohen RA: Relation of brain natriuretic peptide levels to cognitive dysfunction in adults $>55$ years of age with cardiovascular disease. Am J Cardiol 2006;98:538-540.

-26 Gunstad J, Bausserman L, Paul RH, Tate DF, Hoth K, Poppas A, Jefferson AL, Cohen RA: C-reactive protein, but not homocysteine, is related to cognitive dysfunction in older adults with cardiovascular disease. J Clin Neurosci 2006;13:540-546.

27 de Jager CA: Changes over time in memory, processing speed and clock drawing tests help to discriminate between vascular cognitive impairment, mild cognitive impairment and Alzheimer's disease. Neurol Res 2004; 26:481-487.

28 Wentzel C, Rockwood K, MacKnight C, Hachinski V, Hogan DB, Feldman H, Ostbye T, Wolfson C, Gauthier S, Verreault R, McDowell I: Progression of impairment in patients with vascular cognitive impairment without dementia. Neurology 2001;57:714716.

-29 Stanek KM, Gunstad J, Paul RH, Poppas A, Jefferson AL, Sweet LH, Hoth KF, Haley AP, Forman DE, Cohen RA: Longitudinal cognitive performance in older adults with cardiovascular disease: evidence for improvement in heart failure. J Cardiovasc Nurs 2009;24: 192-197.

- 30 Selnes OA, Grega MA, Bailey MM, Pham LD, Zeger SL, Baumgartner WA, McKhann GM: Cognition 6 years after surgical or medical therapy for coronary artery disease. Ann Neurol 2008;63:581-590.
31 Hernandez F Jr, Brown JR, Likosky DS, Clough RA, Hess AL, Roth RM, Ross CS, Whited CM, O'Connor GT, Klemperer JD: Neurocognitive outcomes of off-pump versus on-pump coronary artery bypass: a prospective randomized controlled trial. Ann Thorac Surg 2007;84:1897-1903.

32 Newman MF, Kirchner JL, Phillips-Bute B, Gaver V, Grocott H, Jones RH, Mark DB, Reves JG, Blumenthal JA: Longitudinal assessment of neurocognitive function after coronary-artery bypass surgery. N Engl J Med 2001;344:395-402.

33 Lezak MD, Howieson DB, Loring DW: Neuropsychological Assessment, ed 4. New York, Oxford University Press, 2004.

34 Singer JD, Willett JB: Applied Longitudinal Data Analysis. New York, Oxford University Press, 2003.

35 Guo Z, Viitanen M, Winblad B: Clinical correlates of low blood pressure in very old people: the importance of cognitive impairment. J Am Geriatr Soc 1997;45:701-705.

36 Almeida OP, Flicker L: The mind of a failing heart: a systematic review of the association between congestive heart failure and cognitive functioning. Intern Med J 2001;31:290295.

37 Selnes OA, Grega MA, Borowicz LM Jr, Barry S, Zeger S, Baumgartner WA, McKhann GM: Cognitive outcomes three years after coronary artery bypass surgery: a comparison of on-pump coronary artery bypass graft surgery and nonsurgical controls. Ann Thorac Surg 2005;79:1201-1209.

- 38 Jefferson AL, Tate DF, Poppas A, Brickman AM, Paul RH, Gunstad J, Cohen RA: Lower cardiac output is associated with greater white matter hyperintensities in older adults with cardiovascular disease. J Am Geriatr Soc 2007;55:1044-1048.

39 Roman GC: Brain hypoperfusion: a critical factor in vascular dementia. Neurol Res 2004;26:454-458.

40 Almeida OP, Tamai S: Congestive heart failure and cognitive functioning amongst older adults. Arq Neuropsiquiatr 2001;59:324329.

41 Bennett SJ, Sauve MJ: Cognitive deficits in patients with heart failure: a review of the literature. J Cardiovasc Nurs 2003;18:219-242.

42 Blumenthal JA, Madden DJ, Burker EJ, Croughwell N, Schniebolk S, Smith R, White WD, Hlatky M, Reves JG: A preliminary study of the effects of cardiac procedures on cognitive performance. Int J Psychosom 1991;38:13-16.

43 van Dijk D, Keizer AM, Diephuis JC, Durand C, Vos LJ, Hijman R: Neurocognitive dysfunction after coronary artery bypass surgery: a systematic review. J Thorac Cardiovasc Surg 2000;120:632-639. 
-44 Cook DJ, Huston J 3rd, Trenerry MR, Brown RD Jr, Zehr KJ, Sundt TM 3rd: Postcardiac surgical cognitive impairment in the aged using diffusion-weighted magnetic resonance imaging. Ann Thorac Surg 2007;83: 1389-1395.

-45 Hoth KF, Poppas A, Moser DJ, Paul RH, Cohen RA: Cardiac dysfunction and cognition in older adults with heart failure. Cogn Behav Neurol 2008;21:65-72.

-46 Almeida OP, Tamai S: Clinical treatment reverses attentional deficits in congestive heart failure. BMC Geriatr 2001;1:2

-47 Zuccala G, Onder G, Pedone C, Carosella L, Pahor M, Bernabei R, Cocchi A: Hypotension and cognitive impairment: selective association in patients with heart failure. Neurology 2001;57:1986-1992.

48 Pullicino PM, Hart J: Cognitive impairment in congestive heart failure? Embolism versus hypoperfusion. Neurology 2001;57:19451946.
49 Beer C, Ebenezer E, Fenner S, Lautenschlager NT, Arnolda L, Flicker L, Almeida OP: Contributors to cognitive impairment in congestive heart failure: a pilot case-control study. Intern Med J 2009;39:600-605.

50 Zuccala G, Marzetti E, Cesari M, Lo Monaco MR, Antonica L, Cocchi A, Carbonin P, Bernabei R: Correlates of cognitive impairment among patients with heart failure: results of a multicenter survey. Am J Med 2005; 118: 496-502.

51 Ferrari R, Bachetti T, Agnoletti L, Comini L, Curello S: Endothelial function and dysfunction in heart failure. Eur Heart J 1998; 19(suppl G):G41-G47.

52 Hoth KF, Tate DF, Poppas A, Forman DE, Gunstad J, Moser DJ, Paul RH, Jefferson AL, Haley AP, Cohen RA: Endothelial function and white matter hyperintensities in older adults with cardiovascular disease. Stroke 2007;38:308-312.

53 Selnes OA, Grega MA, Bailey MM, Pham L, Zeger S, Baumgartner WA, McKhann GM: Neurocognitive outcomes 3 years after coronary artery bypass graft surgery: a controlled study. Ann Thorac Surg 2007;84 1885-1896.
54 Shenkin SD, Bastin ME, Macgillivray TJ, Deary IJ, Starr JM, Rivers CS, Wardlaw JM: Cognitive correlates of cerebral white matter lesions and water diffusion tensor parameters in community-dwelling older people. Cerebrovasc Dis 2005;20:310-318.

55 Kalaria RN: Small vessel disease and Alzheimer's dementia: pathological considerations. Cerebrovasc Dis 2002;13(suppl 2): 48-52.

>56 Silbert LC, Nelson C, Howieson DB, Moore MM, Kaye JA: Impact of white matter hyperintensity volume progression on rate of cognitive and motor decline. Neurology 2008; 71:108-113.

57 Jokinen H, Kalska H, Ylikoski R, Madureira S, Verdelho A, van der Flier WM, Scheltens P, Barkhof F, Visser MC, Fazekas F, Schmidt R, O’Brien J, Waldemar G, Wallin A, Chabriat $\mathrm{H}$, Pantoni $\mathrm{L}$, Inzitari $\mathrm{D}$, Erkinjuntti $\mathrm{T}$ : Longitudinal cognitive decline in subcortical ischemic vascular disease - the LADIS Study. Cerebrovasc Dis 2009;27:384-391. 